\title{
Towards a hybrid infrared approach for damage assessment
}

\author{
R. K. Fruehmann ${ }^{\text {a }}$, J. M. Dulieu-Barton ${ }^{\text {b }}$, S. Quinn ${ }^{c}$ \\ University of Southampton, School of Engineering Sciences \\ Southampton, SO17 1BJ, UK \\ arkf@soton.ac.uk, janice@soton.ac.uk, 's.quinn@soton.ac.uk
}

Keywords: Thermoelastic stress analysis (TSA), vibro-thermography (VT), pulsed phase thermography (PPT), non-destructive evaluation (NDE)

\begin{abstract}
The thermoelastic response obtained from an infra-red (IR) detector contains two components: the magnitude of the small stress induced temperature change caused by the thermoelastic effect and the phase angle of the temperature change relative to a reference signal generated by an application of a stress change. The phase angle is related to nonlinearity in the thermoelastic response and departures from the simple linear relationship that underpins thermoelastic stress analysis (TSA). The phase data could be used to make an assessment of temperature evolutions caused by viscoelastic behaviour resulting from damage and provide a basis for its evaluation. In the current paper the physics of other infra-red techniques used for nondestructive evaluation is used to better understand the nature of the thermoelastic response. The objective is to provide better exploitation of TSA by alternative processing of the IR measurements. Three case studies are presented that demonstrate the potential of the alternative processing for evaluating damage.
\end{abstract}

\section{Introduction}

The detection and qualification of damage in engineering materials and structures presents an ongoing challenge. Accordingly there are many tools and many different approaches for assessing damage evolution. One such technique is thermoelastic stress analysis (TSA). The potential for better exploitation of the technique in industrial applications has been demonstrated [1] by modifying the approach to data collection. Placed in the wider context of infra-red (IR) thermography techniques currently used in non-destructive evaluation (NDE), the ultimate aim is to combine pulsed phase thermography (PPT) and acoustic or vibro-thermography (VT) with TSA to enhance NDE procedures. Some initial background studies have been carried out with this in mind [2].

In TSA, PPT and VT, the material or component under inspection is subjected to some form of thermal stimulus, either via an external heat source or via a mechanical input. The resulting evolution of the surface temperature field is analysed to ascertain the location and size of defects within the material. The unique feature of TSA is that it can be used to identify and quantify changes in the stress field due to damage in virtually real time, as the material undergoes cyclic fatigue [1, 3]. A further attractive feature is that, as the technique requires a stress change, measurements can be taken during dynamic cyclic loading, without interrupting the fatigue loading. However, applications to full-scale engineering components are few, e.g. for a wind turbine blade [4]. One reason for the lack of applications in the field is the challenge of applying the technique without the application of a cyclic load. This topic has been examined in [5] and more recently in [1]. To make TSA more effective away from the laboratory, the measured IR signal must be optimised by using alternative means of remote loading (i.e. imparting the temperature change). Techniques for achieving the loading could be drawn from those used with other NDE techniques.

Recent work on fatigue in composite materials has indicated that nonlinearities in the thermoelastic response provide a useful means of identifying damage [6]. As the most notable cause of nonlinearity in the thermoelastic response results from heat transfer, a means of separating the adiabatic and non-adiabatic processes is necessary. This may be achieved by utilising the phase 
of the thermoelastic response. The purpose of the present paper is to provide further insight into the potential for using TSA as a means of quantitative damage assessment. The influence of heat transfer on the magnitude and phase angle of the thermoelastic response is the primary consideration. However, insight into the benefits of a hybrid approach to IR sensing for NDE is also discussed through three case studies. Here TSA data is examined with a focus on considering the non-adiabatic components of the temperature changes. The first two examples focus on the phase data. The third example examines the absolute temperature field as damage evolves under low cycle fatigue.

\section{IR thermography techniques}

In TSA changes in the temperature of a dynamically loaded component are correlated to the changes in the elastic stress at the surface [7]. The technique utilises the reversible change in temperature resulting from an elastic strain. The amplitude of the change in temperature is directly proportional to the amplitude of the change in stress. Typically, a sinusoidal load is applied, producing a corresponding sinusoidal response in the surface temperature that can be filtered from the IR signal and the amplitude measured with a precision to the order of $\mathrm{mK}$. The IR signal is processed to extract the reversible components of the temperature change at the surface of the material. Two additional data-sets are also produced: the mean temperature field and the phase angle of the temperature change relative to a reference signal generated by a stress change. If the phase angle is zero (or $180^{\circ}$ ) then the thermoelastic temperature change is occurring at the same instance as the stress change, i.e. in phase and the response is isentropic. If it is not then the response is nonlinear as a result of a combination of effects, the most likely being heat transfer. Wang et al [8] provide an illustration of the effect of non adiabatic behaviour. The mean temperature field can also be used to assess irreversible heat generation, for example, due to frictional or viscoelastic heating. It is in the interpretation of these two data sets that concepts from PPT and VT can be exploited.

In PPT an external heat pulse is used to introduce a temperature change at the surface of a material. The pulse is transmitted through the material as a wave containing many frequency components. When the wave meets a discontinuity, for example a region of damage, the wave is reflected causing a temperature change at the surface. The depth of the defect is obtained using the phase angle of the reflected wave, while the size of the defect can be measured by its spatial extent of data with the same phase angle $[9,10]$. Depending on the conductivity of the material and the magnitude and frequency of the thermal pulse, individual frequency components are propagated and reflected in different ways. Lower frequency components can propagate further into the material, while higher frequency components are attenuated more quickly but allow smaller defects to be identified.

In VT, an ultrasonic wave is used to excite the material. In regions of damage, frictional and viscoelastic effects cause local heating. The generated heat is transmitted to the surface of the material where defects result in regions of elevated temperature. The depth of defect that can be detected depends on the magnitude of the energy release rate at the defect, the duration of the excitation and the conductivity of the material [11]. Successful application of the technique is crucially dependent on the ability to introduce an ultrasonic wave in such a manner that existing damage is not propagated further and that new damage is not introduced at the point of excitation. This must be balanced with the need for an excitation of sufficient magnitude to enable the existing damage to be identified.

In TSA the material can be treated as a periodic heat source. Non-uniformities result in a gradient in the amplitude of the local heat sources, and hence lead to heat transfer effects, similar to those exploited in PPT and VT. The two important concepts from PPT and VT that may be of interest in TSA are periodic temperature changes being transmitted through the material as a thermal wave, and the use of localised heating as a means of locating damage. 


\section{Case studies}

Figure 1 shows the full set of TSA data taken from the neighbourhood of a $30^{\circ}$ slot in a thin aluminium plate. The plate is undergoing tension-tension cyclic loading and as a result a cracks are propagating from each slot tip transversely to the loading direction. In this data the load amplitude was maintained at a low level to avoid further growth of the crack during the data collection. From the mean temperature across the plate (Figure 1a), it is evident that the mean temperature field is uniform indicating that the damage is not leading to localised heating. The TSA data, $\Delta T$ (Figure 1b) clearly shows two large stress concentrations either side of the slot. However, the exact position of the crack tip, and the exact magnitude of the stress concentration are difficult to identify from the $\Delta T$ data. This is due to heat transfer near the crack tip where the stress induced temperature gradients are large. The effect of heat transfer can be seen clearly in the phase data (Figure 1c). Taking a line of data through the upper stress concentration (line $y=108$ ) allows the deviations in the phase angle to be correlated to the $\Delta T$ data (Figure $1 \mathrm{~d}$ ). The dotted line (left $\mathrm{y}-$ axis) shows the TSA data; the crosses (right hand y-axis) show the phase angle. The position of the
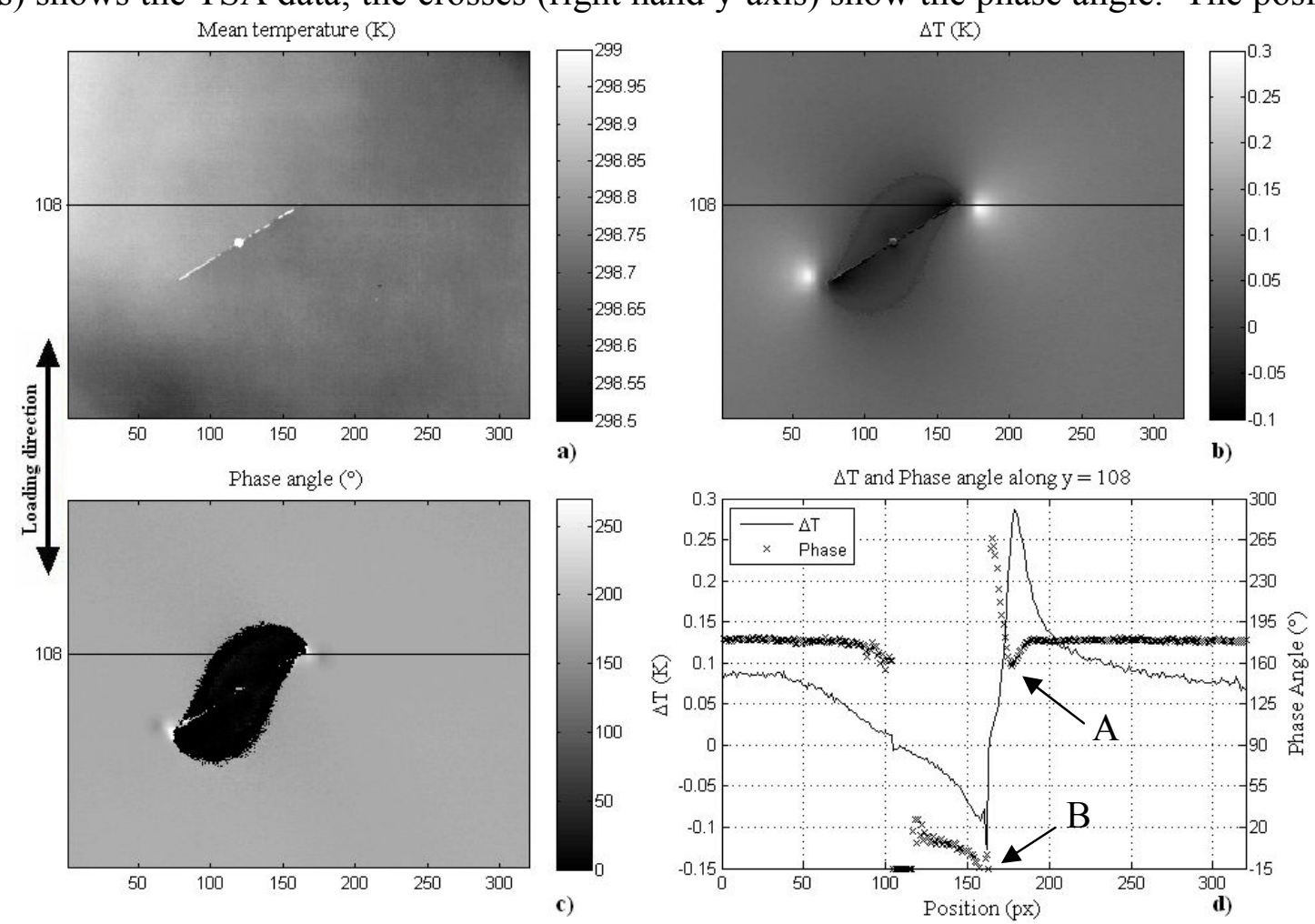

Figure 1: TSA data from a $30^{\circ}$ slot in a thin aluminium plate

local phase minimum (point A in Figure 1d) was used by Zanganeh et al [12] to identify the location of the crack tip. This minimum occurs due to heat conduction away from the region of highest $\Delta T$ and is best understood by considering the stress concentration as a point source generating a thermal wave. As the wave propagates away from the source, it results in a thermal oscillation in the adjacent region with a time delay dependent on the conductivity of the material, which occurs over about 10 pixels (i.e. $5 \mathrm{~mm}$ ). The phase angle departs from $180^{\circ}$ indicating that heat transfer is taking place as a result of the large stress/thermal gradient adjacent to the crack tip. The transition from tensile to compressive stress to the left of point A shows first an increase in phase to around $260^{\circ}$ before a step change to $0^{\circ}$ to the left of point $\mathrm{B}$. The stress is close to zero in the region between points $\mathrm{A}$ and $\mathrm{B}$ where the crack line is crossed just above the tip. Here the $\Delta T$ readings result only as a consequence of heat transfer.

Very similar phenomena can be seen in woven composite materials undergoing high cycle fatigue loading. The data shown in Figure 2 is taken from a 2 × 2 twill woven E-glass / epoxy, single ply composite specimen loaded in tension. The weave pattern is superimposed on the images, with the warp yarns running parallel to the loading direction. A transverse crack (circled in 
Figure $2 \mathrm{a}, \mathrm{b}$ and $\mathrm{c})$ is present in one weft yarn. Again, the TSA and phase data from a line $(\mathrm{y}=40)$ drawn through the damaged region has been plotted in the bottom right image in Figure 2. The phase data in the vicinity of the damage in the woven composite material shows similar trends to those from the aluminium plate, despite the non-uniform $\Delta T$ field; there is a reduction in the phase angle close to the crack tip because of the large stress gradients in the damaged region. In [6] it was demonstrated that the phase data thereby enables damage to be detected within a noisy / nonuniform $\Delta T$ field.

An additional feature seen in the TSA data in Figure 2, is a $15^{\circ}$ difference in phase between the tows running parallel and transverse to the loading direction. The warp yarns exhibit the positive phase shift indicating heat transfer to the warp yarns. In the TSA data it can be seen that the weft yarns have the larger thermoelastic response, despite the majority of the stress being carried by the warp yarns [13]. In this case, a stress gradient is occurring through the thickness of a material, rather than in the plane. The surface phase shift therefore identifies a subsurface stress
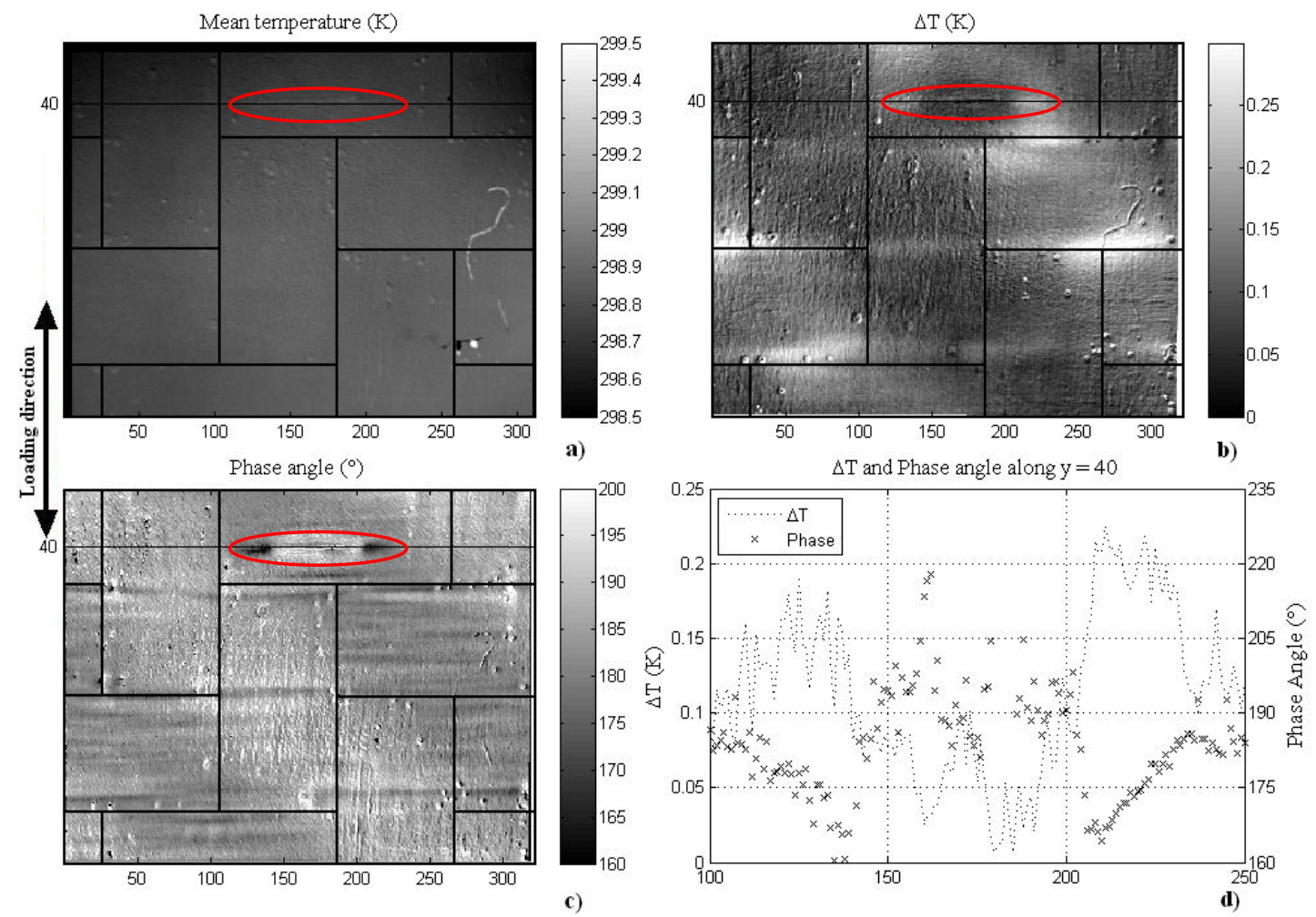

Figure 2: TSA phase images from a 2 × 2 twill woven, single ply composite

concentration, the magnitude of the phase shift depending on the thermal conductivity of the material and the loading frequency. This idea has been used in [14] and links in with ideas from PPT, where a thermal pulse consisting of a summation of thermal waves of different frequencies and magnitudes is introduced into the material. By decomposing the thermal wave as it reaches the surface it is possible to use the phase of the different frequency components to obtain information regarding a range of sizes and depths of defects. This idea could equally be applied to TSA data. Instead of using only a single excitation frequency (the loading frequency), random loading, or loading with multiple simultaneous frequencies may provide a more useful thermoelastic response to maximise the information obtained from the phase data. This idea could be applied to the study of components during in-service loading. Alternatively, a single transient step load could be applied, generating a square wave which comprises multiple frequencies, as in PPT.

Figure 3 shows 250 frames from a 1050 frame recording taken at $101 \mathrm{~Hz}$ of the temperature-time history from three areas on an E-glass epoxy composite strip specimen with a cross ply $[90,0]_{2 \mathrm{~S}}$ layup. A $2.2 \mathrm{~mm}$ long, $0.7 \mathrm{~mm}$ wide notch has been cut into its right hand edge and cyclic tensile loading applied. Figure 3 also shows the corresponding TSA data, i.e. the amplitude of the temperature shown in the graph, but calculated using only the data collected after frame 300 . 


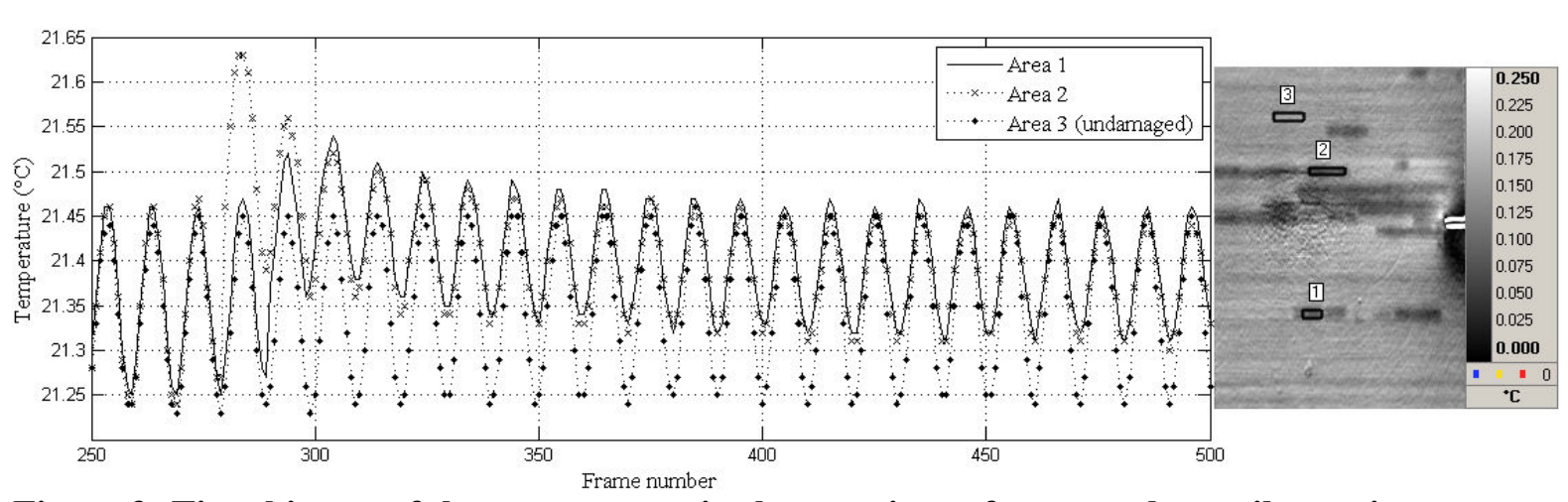

Figure 3: Time history of the temperature in three regions of a cross-ply tensile specimen

Damage growth can be observed in the $90^{\circ}$ surface ply, away from the notch region in areas 1 and 2. Area 3 shows a region that does not sustain damage during the measurement period. The significant feature in this data is the sharp increase in temperature at the instant the damage occurs which can be linked to the energy released as a crack forms. The increase in temperature quickly dissipates, and the amplitude of the temperature signal is reduced as the stress is redistributed to the $0^{\circ}$ ply below. (The small decrease in the mean temperature between frames 220 and 230 can be discounted as a background reflection.)

Filtering out the $10 \mathrm{~Hz}$ frequency shows the non-adiabatic temperature changes occurring in the material, shown in Figure 4. A significant difference between the temperature profiles of area 1 and 2 can be seen in the rise time of the peak at frame 280. While in area 2 there is an almost instantaneous rise to the peak temperature, in area 1 the rise takes place over the course of approximately twenty frames $(0.2$ seconds $)$. Two explanations are proposed for the difference in

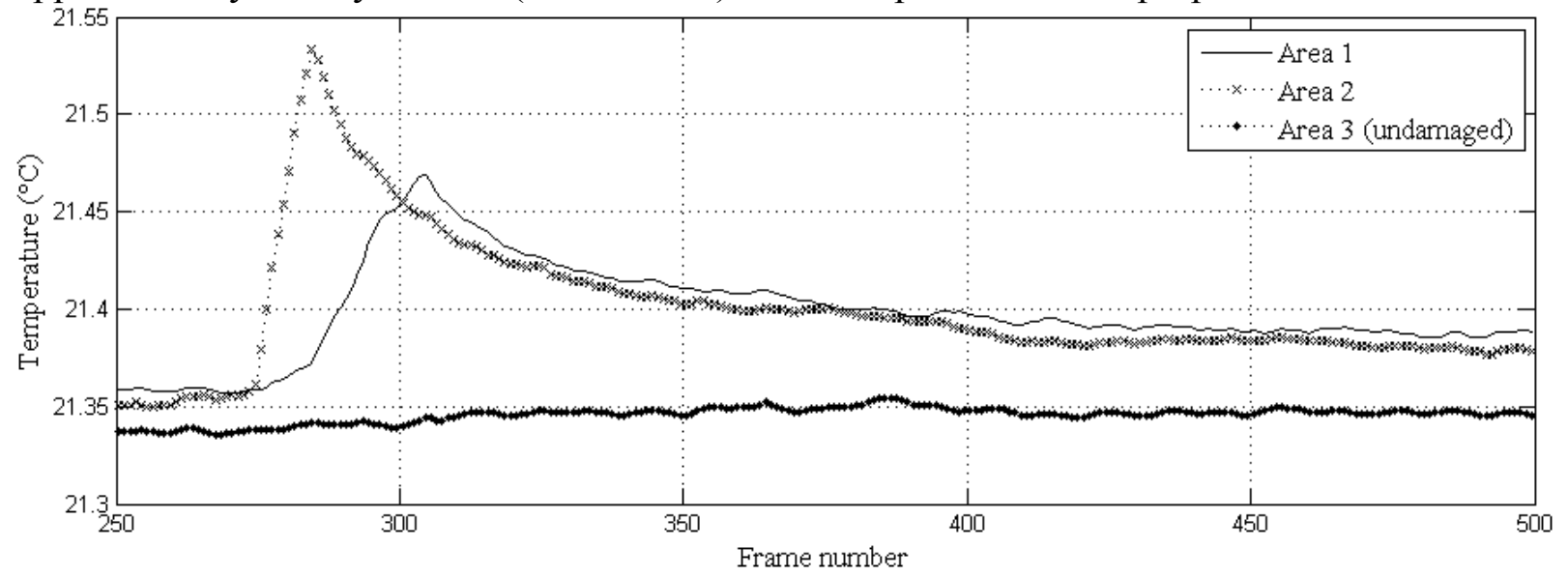

Figure 4: Mean temperature of areas 1, 2 and 3 from Figure 3

the response in the two areas. Firstly, area 1 could contain a subsurface crack and therefore a time lag would be expected as the heat travels to the surface or, secondly, fibre bridging might be occurring as the crack forms, slowing the rate of growth and dissipating energy over a longer time. The area under the curves could be used to estimate the energy released during the damage event.

\section{Conclusions}

It is has been shown that the phase of the thermoelastic response can be exploited to derive more detailed information regarding the depth of defects and the nature of subsurface damage. It has been suggested that similar ideas to those used in PPT would enable the application of the phase data in a quantitative manner. The most interesting aspect of this is the potential for evaluating components under in-service loading, where multiple frequency loading could be used as an advantage over conventional sinusoidal loading used in laboratory work. In this context, alternative loading scenarios for laboratory testing of materials and components may also be developed. 
It has also been shown that the change in temperature as energy is released during the formation and growth of cracks can be obtained from the IR measurements. To fully exploit this aspect, suitable algorithms for identifying the energy release event are required. These could be used to trigger a TSA measurement from which the influence of the damage on the stress field can be evaluated.

The paper shows how a holistic approach towards IR measurement has the potential to provide a powerful tool for strain based NDE of engineering materials and structures.

\section{References}

[1] R.K. Fruehmann, J.M. Dulieu-Barton and S. Quinn: Experimental Mechanics, (2009), DOI: 10.1007/s11340-009-9295-9

[2] T.R. Emery and J.M. Dulieu-Barton: Key Engineering Materials Vol. 347 (2007), p. 621

[3] T.R. Emery and J.M. Dulieu-Barton: Composites Part A (2009), DOI: 10.1016/j.compositesa.2009.08.015

[4] R. J. H. Paynter and A.G. Dutton: Strain Vol 39 (2003), p. 73

[5] U. Galietti, D. Modugno and L. Spagnolo: Measurement Science and Technology Vol 16 (2005), p. 2251

[6] R.K. Fruehmann, J.M. Dulieu-Barton and S. Quinn in: Proceedings of ICCM 17 (2009), on cd

[7] J. M. Dulieu-Barton and P. Stanley: Journal of Strain Analysis Vol. 33 (1998), p. 93

[8] W.J. Wang, J.M. Dulieu-Barton and Q. Li : Experimental Mechanics (2009), Vol. 50, p 449. DOI: $10.1007 / \mathrm{s} 11340-009-9249-2$

[9] X. P. Maldague and S. Marinetti: Journal of Applied Physics Vol 79(1996), p. 2694

[10]G. Busse, D. Wu and W. Karpen: Journal of Applied Physics Vol 71 (1992), p. 3962

[11]T.J. Barden, D.P. Almond, S.G. Pickering, M. Morbidini and P. Cawley: Nondestructive Testing and Evaluation Vol 22 (2007), p. 71

[12]M. Zanganeh, R.A. Tomlinson and J.R. Yates: Journal of Strain Analysis Vol: 43 (2008), p. 529

[13] R.K. Fruehmann, J.M. Dulieu-Barton and S. Quinn: Journal of Strain Analysis Vol: 43 (2008), p. 435

[14]N. Sathon and J.M. Dulieu-Barton: Applied Mechanics and Materials Vol: 7-8 (2007), p. 153 